\title{
Adesão ao tratamento de hanseníase em pacientes com episódios reacionais hansênicos em uma unidade de referência
}

Adherence to treatment of leprosy in patients with leprosy reactional episodes in a reference unit

Adhesión al tratamiento de la hanseníasis en pacientes con episodios de reacción hansénica en una unidad de referencia

Maria de Fátima Silveira Abraçado

Universidade Federal do Pará, Belém, Pará, Brasil

Maria Heliana Chaves Monteiro da Cunha

Núcleo de Medicina Tropical, Universidade Federal do Pará, Belém,

Pará, Brasil
Marília Brasil Xavier

Núcleo de Medicina Tropical, Universidade Federal do Pará, Belém,

Pará, Brasil

Universidade Estadual do Pará, Belém, Pará, Brasil

\section{RESUMO}

Neste artigo é avaliada a interferência de fatores sociodemográficos e clínicos na adesão ao tratamento de portadores de reação hansênica em uma unidade de referência dermatológica da Região Metropolitana de Belém, Estado do Pará, Brasil, no período de 2005 a 2010. Trata-se de um estudo transversal, de cunho descritivo, em uma amostra de 214 indivíduos em tratamento de reação hansênica. Os resultados mostraram predominância de adultos do sexo masculino com baixa escolaridade, maior incidência da forma clínica dimorfa e tratamento multibacilar. O tipo de reação predominante foi a reação reversa. $O$ maior percentual dos pacientes apresentou de um a três episódios reacionais, acompanhados de neurite. A associação entre fatores sociodemográficos e adesão apontou o sexo masculino com três vezes mais chances de não aderir ao tratamento $(p<0,0110)$. Não houve associação entre adesão e variáveis clínicas. O índice de adesão ao tratamento foi considerado elevado.

Palavras-chave: Adesão à Medicação; Hanseníase; Efeitos Colaterais; Reações Adversas Relacionadas a Medicamentos.

\section{INTRODUÇÃO}

A principal sequela da hanseníase é a incapacidade física, que pode resultar de um diagnóstico tardio e de tratamento inadequado, ou ser produzida por episódios reacionais hansênicos sucessivos (reentrantes), os quais são respostas exacerbadas do sistema imunológico ao agente agressor. Esses episódios, se não forem tratados de forma adequada, de acordo com as normas do Ministério da Saúde (MS), poderão levar a incapacidades físicas pelo comprometimento neural ${ }^{1,2,3,4}$.

Existem dois tipos de reação hansênica: a reversa (RR) ou tipo 1, que é uma resposta exacerbada do sistema imunológico do tipo celular, própria de

\footnotetext{
Correspondência / Correspondence / Correspondencia:

Maria de Fátima Silveira Abraçado

Av. Rômulo Maiorana, $n^{\circ} 74$, bloco A, ap. 202. Bairro: Marco

CEP: 66093-635 Belém-Pará-Brasil

Tel.: +55 (91) 3236-1960/98336-1105

E-mail: fatimaabracado@gmail.com
}

portadores das formas tuberculoide e dimorfa, cujo quadro clínico é caracterizado por sinais e sintomas de inflamação como dor, eritema, infiltração e edema de lesões cutâneas pré-existentes, ou, raramente, acompanhado de novas lesões; e a reação tipo 2 ou eritema nodoso, que traduz uma hipersensibilidade da imunidade do tipo humoral, acomete portadores das formas virchowiana e dimorfa-virchowiana ${ }^{5}$, e se caracteriza por neurite, cujos sintomas são espessamento de um ou mais nervos periféricos, seguido de dor e acentuada alteração da sensibilidade no trajeto neural, e, dependendo da gravidade, pode levar à formação do impropriamente chamado "abscesso neural" 16,7 .

Poucos trabalhos abordam o problema da falta de adesão ao tratamento da hanseníase e procuram esclarecer as causas desta irregularidade, principalmente quando da presença das reações hansênicas. Neste sentido, este estudo objetiva identificar a presença de fatores clínicos e não clínicos (sociais e demográficos) que poderiam estar associados à adesão ao tratamento das reações hansênicas em uma Unidade de Referência no Estado do Pará. 


\section{MATERIAIS E MÉTODOS}

Trata-se de um estudo do tipo transversal, de cunho descritivo, realizado em pacientes com diagnóstico de hanseníase que apresentaram reação hansênica em acompanhamento ambulatorial na Unidade de Referência Dr. Marcelo Cândia, Região Metropolitana de Belém, no período de 2005 a 2010.

$\bigcirc$ estudo foi realizado em 214 indivíduos, de ambos os sexos e de todas as idades, portadores de hanseníase, que desenvolveram reação hansênica. Foram excluídos do estudo pacientes que apresentaram recidivas, além dos pacientes com prontuários que não continham informações suficientes para o desfecho da pesquisa.

Os dados foram coletados nos prontuários da unidade de referência, por meio de um formulário contendo variáveis sociodemográficas como: sexo, idade, procedência e tipo de ocupação; e variáveis clínicas do tipo: classificação clínica da hanseníase, tipo de episódios reacionais, incidência de casos reacionais ao ano, sintomatologia específica das reações, medicações prescritas e frequência nos dias aprazados.

A análise estatística foi realizada no programa BioEstat 5.0. Os testes estatísticos aplicados nas análises bivariadas foram o qui-quadrado, o teste $G$ e o teste exato de Fisher, considerando p-valor $\leq 0,05$ e intervalo de confiança $95 \%$.

Os critérios utilizados para adesão ao tratamento de reações neste trabalho foram:

- Adesão ao tratamento: pacientes que tomaram a medicação regularmente e pelo tempo necessário preconizado para a regressão das lesões, com menos de três faltas consecutivas às consultas agendadas, ou $75 \%$ de comparecimento às consultas agendadas; e

- Não adesão ao tratamento: pacientes com três ou mais faltas consecutivas às consultas agendadas ou frequência inferior a $75 \%$.

O diagnóstico clínico para hanseníase adotado pelos dermatologistas do Centro de Referência Dr. Marcelo Cândia segue os padrões da classificação de Madri: indeterminada (I), dimorfa (D), tuberculoide (T) e virchowiano $(V)^{1,3}$, e do $M S$, por meio da classificação operacional PB para os paucibacilares e MB para os multibacilares. Os pacientes que desenvolveram reação hansênica foram classificados, de acordo com os aspectos clínicos apresentados, em portadores de reação tipo 1 e de reação tipo 2, e tratados conforme as normas do MS para cada caso.

A pesquisa foi aprovada pelo Comitê de Ética da Fundação Hospital de Clínicas Gaspar Vianna, com n de protocolo 250/2010 em 15 de dezembro de 2010 e autorizada pela direção da Unidade de Referência Dr. Marcelo Cândia.

\section{RESULTADOS}

Constatou-se que 0 tratamento das reações hansênicas nos anos analisados neste estudo apresentou variação entre adesão e não adesão. Dessa maneira, os anos de 2006, 2009 e 2010 apresentaram predomínio de adesão ao tratamento; por outro lado, os anos de 2005, 2007 e 2008 demonstraram maior não adesão (Figura 1).

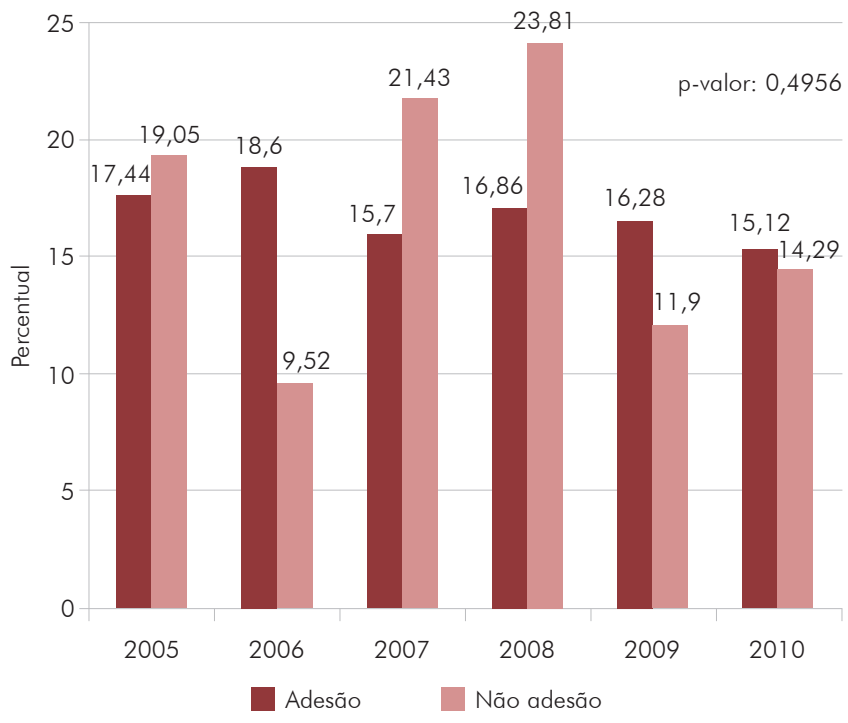

Figura 1 - Distribuição dos pacientes de acordo com a adesão ao tratamento das reações, 2005-2010

Considerando as características sociodemográficas dos pacientes estudados (Tabela 1), constatou-se que o sexo possui associação estatisticamente significativa com o fato de aderir ou não ao tratamento, tendo os homens, aproximadamente, três vezes mais chances de não aderir ao tratamento do que as mulheres. As demais características não apresentaram associação estatisticamente significativa com o fato de aderir ou não ao tratamento; contudo, descritivamente pode-se dizer que os pacientes da faixa etária de 29 a 49 anos, separados/viúvos/solteiros, foram os que mais aderiram ao tratamento. $\bigcirc$ nível escolar até o ensino fundamental apresentou um percentual de não adesão de $73,8 \%$.

Não houve nenhuma relação estatisticamente significativa entre características clínicas e o fato de aderir ou não ao tratamento (Tabela 2). Contudo, descritivamente, pode-se dizer que houve uma diferença percentual pequena entre as taxas de adesão $(61,34 \%)$ e não adesão $(60,61 \%)$ entre os que tinham mais de um ano de diagnóstico da doença. Os pacientes diagnosticados como MB $(87,79 \%)$, com presença de neurites $(77,91 \%)$, número de episódios igual ou superior a três $(89,53 \%)$ e com tipo de reação RR $(80,23 \%)$ também apresentaram maior percentual de adesão. 
Tabela 1 - Distribuição dos pacientes de acordo com as características sociodemográficas e a adesão ao tratamento das reações, 2005-2010

\begin{tabular}{|c|c|c|c|c|c|c|c|c|}
\hline \multirow{2}{*}{ Variável } & \multicolumn{2}{|c|}{ Adesão } & \multicolumn{2}{|c|}{ Não adesão } & \multicolumn{2}{|c|}{ Total } & \multirow{2}{*}{ Teste estatístico $p$-valor } & \multirow{2}{*}{ O.R. (p-valor) } \\
\hline & $\mathrm{N}$ & $\%$ & $N$ & $\%$ & $\mathrm{~N}$ & $\%$ & & \\
\hline Gênero & & & & & & & Qui-quadrado & \\
\hline Feminino & 67 & 38,9 & 7 & 16,7 & 74 & 34,6 & & \\
\hline Masculino & 105 & 61,1 & 35 & 83,3 & 140 & 65,4 & $0.0110^{*}$ & 3.1905 (0.01 10) \\
\hline Total & 172 & 100,0 & 42 & 100,0 & 214 & 100,0 & & \\
\hline Faixa etária & & & & & & & Teste $G$ & \\
\hline Até 15 anos & 13 & 7,6 & 2 & 4,8 & 15 & 7,0 & & \\
\hline 16 a 25 anos & 40 & 23,3 & 13 & 31,0 & 53 & 24,8 & & \\
\hline 26 a 49 anos & 71 & 41,2 & 12 & 28,6 & 83 & 38,8 & 0.3545 & - \\
\hline 50 ou mais anos & 48 & 27,9 & 15 & 35,6 & 63 & 29,4 & & \\
\hline Total & 172 & 100,0 & 42 & 100,0 & 214 & 100,0 & & \\
\hline Estado civil & & & & & & & Qui-quadrado & \\
\hline Separado(a) / viúvo(a) / solteiro(a) & 87 & 50,6 & 20 & 47,6 & 107 & 50,0 & & \\
\hline União estável/casado & 85 & 49,4 & 22 & 52,4 & 107 & 50,0 & 0.8633 & $1.1259(0.8633)$ \\
\hline Total & 172 & 100,0 & 42 & 100,0 & 214 & 100,0 & & \\
\hline Nível de escolaridade & & & & & & & Qui-quadrado & \\
\hline Até o Ensino Fundamental & 129 & 75,0 & 31 & 73,8 & 160 & 74,8 & & \\
\hline A partir do Ensino Médio & 43 & 25,0 & 11 & 26,2 & 54 & 25,2 & 0.9690 & $1.0645(0.9690)$ \\
\hline Total & 172 & 100,0 & 42 & 100,0 & 214 & 100,0 & & \\
\hline Renda familiar & & & & & & & Qui-quadrado & \\
\hline < 1 salário mínimo & 49 & 28,5 & 12 & 28,6 & 61 & 28,5 & & \\
\hline > 1 salário mínimo & 123 & 71,5 & 30 & 71,4 & 153 & 71,5 & 0.3536 & $1.4939(0.3536)$ \\
\hline Total & 172 & 100,0 & 42 & 100,0 & 214 & 100,0 & & \\
\hline
\end{tabular}

* Nível descritivo ( $p$-valor) estatisticamente significativo ao nível de significância $\alpha=0.05$. Sinal convencional utilizado: - Dado numérico igual a zero não resultante de arredondamento.

Tabela 2 - Distribuição dos pacientes de acordo com as características clínicas e a adesão ao tratamento, 2005 a 2010

\begin{tabular}{|c|c|c|c|c|c|c|c|c|}
\hline \multirow{2}{*}{ Variável } & \multicolumn{2}{|c|}{ Adesão } & \multicolumn{2}{|c|}{ Não adesão } & \multicolumn{2}{|c|}{ Total } & \multirow{2}{*}{ Teste estatístico p-valor } & \multirow{2}{*}{ O.R. (p-valor) } \\
\hline & $\mathrm{N}$ & $\%$ & $\mathrm{~N}$ & $\%$ & $\mathrm{~N}$ & $\%$ & & \\
\hline Tempo de diagnóstico & & & & & & & Qui-quadrado & \\
\hline$<1$ ano & 46 & 38,66 & 13 & 39,39 & 59 & 38,82 & & \\
\hline$>1$ ano & 73 & 61,34 & 20 & 60,61 & 93 & 61,18 & $X^{2}=0.9007$ & $0.9694(0.9007)$ \\
\hline Total & 119 & 100,0 & 33 & 100,0 & 152 & 100,0 & & \\
\hline Tipo de tratamento & & & & & & & Qui-quadrado & \\
\hline Multibacilar & 151 & 87,79 & 35 & 83,33 & 186 & 86,92 & & \\
\hline Palcibacilar & 21 & 12,21 & 7 & 16,67 & 28 & 13,08 & $X^{2}=0.6081$ & $1.4381(0.6081)$ \\
\hline Total & 172 & 100,0 & 42 & 100,0 & 214 & 100,0 & & \\
\hline Neurites & & & & & & & Qui-quadrado & \\
\hline Não & 38 & 22,09 & 8 & 19,05 & 46 & 21,50 & & \\
\hline $\operatorname{Sim}$ & 134 & 77,91 & 34 & 80,95 & 168 & 78,50 & $X^{2}=0.8249$ & $1.2052(0.8249)$ \\
\hline Total & 172 & 100,0 & 42 & 100,0 & 214 & 100,0 & & \\
\hline$N^{\circ}$ de episódios reacionais & & & & & & & Exato de Fisher & \\
\hline$\geq 3$ & 154 & 89,53 & 37 & 88,10 & 191 & 89,25 & & \\
\hline$<3$ & 18 & 10,47 & 5 & 11,90 & 23 & 10,75 & 0.7820 & $1.1562(0.9938)$ \\
\hline Total & 172 & 100,0 & 42 & 100,0 & 214 & 100,0 & & \\
\hline Tipo de reação & & & & & & & Teste G & \\
\hline Eritema nodoso hansênico & 6 & 3,49 & - & - & 6 & 2,80 & & \\
\hline Reação reversa & 138 & 80,23 & 40 & 95,24 & 178 & 83,18 & $00312 *$ & \\
\hline Reação reversa/Eritema nodoso hansênico & 28 & 16,28 & 2 & 4,76 & 30 & 14,02 & 0.0312 & - \\
\hline Total & 172 & 100,0 & 42 & 100,0 & 214 & 100,0 & & \\
\hline
\end{tabular}




\section{DISCUSSÃO}

Apesar do impacto econômico e social que as reações hansênicas produzem na vida dos indivíduos acometidos por essa intercorrência, estudos a respeito da adesão ao tratamento da mesma ainda são escassos.

A caracterização sociodemográfica da população estudada mostrou predominância de adultos do sexo masculino, com baixa escolaridade, forma autônoma de trabalho, com renda familiar entre um e dois salários mínimos, oriundos da Região Metropolitana de Belém e da região nordeste paraense, as quais são consideradas áreas de alta endemicidade em hanseníase ${ }^{8}$. A hanseníase, em geral, concentra-se em locais de maior pobreza, estreitando relação com precárias habitações, baixa renda e baixa escolaridade, o que favorece a difusão da doença ${ }^{9}$.

Adultos de baixa renda e baixa escolaridade são achados comuns nos trabalhos epidemiológicos de hanseníase; esses indivíduos, em pleno vigor laboral, em virtude da baixa escolaridade, normalmente já têm dificuldade em conseguir trabalho; some-se a isso o quadro reacional hansênico, que aumenta muito mais as dificuldades, requerendo que a equipe de saúde tenha um olhar mais cuidadoso para esses casos. Aquino et $a^{10}$ e Pimentel ${ }^{11}$, em suas pesquisas, também encontraram resultados semelhantes.

Chama a atenção, neste estudo, o percentual de crianças e de adolescentes que trataram hanseníase: apesar de baixo para as demais variáveis, é relevante uma vez que demonstra a existência de focos ativos da doença, provavelmente sem tratamento ${ }^{12}$. Para um serviço de hanseníase e diante deste achado que condiz com a realidade endêmica do Estado do Pará, em relação aos menores de 15 anos de idade ${ }^{8}$, urge realizar uma vigilância epidemiológica mais efetiva sobre os casos de hanseníase e seus contatos intradomiciliares.

As características clínicas da hanseníase demonstraram predomínio das formas consideradas multibacilares, concordantes com o tipo de tratamento adotado. Este achado é coerente para uma área endêmica, como é o Estado do Pará. A Coordenação do Programa Nacional de Controle da Hanseníase (PNCH/MS) ${ }^{8,12}$ divulgou o comprometimento da Região Amazônica em relação à concentração de casos novos de doença perante o restante do país: 38,9\% (15.532 casos), ocupando o Estado do Pará o quinto lugar na taxa de detecção de casos novos, 62,17\%. Outros estudos também apontam a Região Norte como uns dos clusters da doença na Amazônia ${ }^{8,12}$.

A RR é a que gera maiores danos orgânicos e sociais, pela característica de produzir quadro agudo com dor e pela possibilidade de dano neural, incapacitando o portador para o trabalho, levando a licenças médicas recorrentes e consequente desemprego $6,7,13,14$.

Neste estudo, a análise da influência dos fatores sociodemográficos na adesão ao tratamento das reações hansênicas mostrou que o sexo masculino tem três vezes mais chances de não aderir ao tratamento do que o feminino. Historicamente a mulher sempre teve maior disponibilidade e acessibilidade em comparecer às unidades de saúde, seja para tratar sua saúde ou a de seus familiares; o homem sempre apresentou barreiras nesse sentido, com justificativas apoiadas na jornada de trabalho. Outros estudos também encontraram no sexo masculino maior dificuldade em frequentar os serviços de saúde ${ }^{15,16}$. Desse modo, convém às unidades de saúde, que tratam portadores de hanseníase, enfatizar a necessidade de manter assiduidade às consultas, principalmente dos pacientes do sexo masculino, e, principalmente, alertá-los sobre o aparecimento das reações hansênicas e suas consequências.

É importante destacar que os pacientes deste estudo têm, em sua maioria, baixa escolaridade. Este fator deve ser observado com atenção no momento de verbalizar orientações e esclarecimentos a respeito da hanseníase e das reações hansênicas. Alguns estudos na Índia e outros no Brasil, como o de Aquino et $a^{10}$, correlacionam a importância da educação básica e a melhoria do nível de compreensão do indivíduo acerca da doença e ressaltam este fator para a adesão ao tratamento.

Os resultados do estudo, relacionando características clínicas com adesão, apesar de não apresentarem nenhuma relação com significância estatística, apontaram maior tendência à adesão de pacientes com formas multibacilares, com tempo de diagnóstico de hanseníase maior que um ano, tipo reacional $R R$ e neurites. Esta tendência se constitui em fato positivo para a unidade em que foi realizado o estudo, uma vez que o tratamento multibacilar é longo, a quantidade de drogas a ser ingerida é bem maior, podendo ocorrer efeitos colaterais. Outros trabalhos apontaram maior tendência em não aderir ao tratamento em pacientes com essas características clínicas, com justificativas para a não adesão de que o tratamento é muito "longo e difícil"14,15.

A análise da adesão ao tratamento, apesar de mostrar taxa percentual positiva de adesão, alerta para a existência de taxas de não adesão que, olhadas anualmente, são preocupantes em virtude das consequências físicas severas geradas por danos neurais reversíveis e até irreversíveis; e também consequências sociais, como desemprego e discriminação, entre outras.

\section{CONCLUSÃO}

Neste estudo constatou-se que as reações hansênicas se manifestaram em maior número durante o período de tratamento da hanseníase. A análise da associação de fatores sociodemográficos na adesão ao tratamento mostrou o sexo masculino com três vezes mais chances de não aderir ao tratamento que o feminino. Não houve interferência de fatores clínicos na adesão. A adesão ao tratamento em pacientes com reação hansênica na Unidade de Referência mostrou índice elevado. 


\title{
Adherence to treatment of leprosy in patients with leprosy reactional episodes in a reference unit
}

\begin{abstract}
In this study, it was evaluated the interference of socio-demographic and clinical factors in the adherence to treatment in patients with leprosy reaction in a dermatology unit of reference in the Metropolitan Region of Belém, Pará State, Brazil, during the period from 2005 to 2010. It was a cross-sectional descriptive study in a sample of 214 individuals in treatment of leprosy reaction. The results showed a predominance of adult males with low education, higher incidence of dimorphous leprosy and multibacillary treatment. The predominant reaction was the reverse reaction. The higher percentage of patients was from one to three reactional episodes with neuritis. The connection between socio-demographic factors and that adherence pointed male sex with three times more likely to not accept the treatment $(p<0.0110)$.
\end{abstract}

Keywords: Medication Adherence; Leprosy; Side Effects; Drug Adverse Reactions.

\section{Adhesión al tratamiento de la hanseníasis en pacientes con episodios de reacción hansénica en una unidad de referencia}

\section{RESUMEN}

En este artículo se evalúa la interferencia de factores sociodemográficos y clínicos en la adhesión al tratamiento de portadores de reacción hansénica en una unidad de referencia dermatológica de la Región Metropolitana de Belém, Estado de Pará, Brasil, en el período de 2005 a 2010. Se trata de un estudio transversal, de cuño descriptivo, en una muestra de 214 individuos en tratamiento de reacción hansénica. Los resultados mostraron predominancia de adultos de sexo masculino con baja escolaridad, mayor incidencia de la forma clínica dimorfa y tratamiento multibacilar. El tipo de reacción predominante fue la reacción reversa. El mayor porcentaje de los pacientes presentó de un a tres episodios reactivos, acompañados de neuritis. La asociación entre factores sociodemográficos y adhesión señaló que el sexo masculino tiene tres veces más oportunidades de no adherir al tratamiento $(p<0,0110)$. No hubo asociación entre adhesión y variables clínicas. El índice de adhesión al tratamiento fue considerado elevado.

Palabras clave: Cumplimiento de la Medicación; Lepra; Efectos Colaterales; Reacciones Adversas Relacionados con Medicamentos.

\section{REFERÊNCIAS}

1 Talhari S, Neves RG, Oliveira MLW, Andrade ARC, Ramos AMC, Penna GO, et al. Manifestações cutâneas e diagnóstico diferencial. In: Talhari S, Neves RG, Penna GO, Oliveira MLV, editores. Hanseníase. 4. ed. Manaus: Tropical; 2006. p. 21 58.

2 Jacobson RR, Krahenbuhl JL. Leprosy. Lancet. 1999 Feb;353(9153):655-60.

3 Opromolla DVA. Noções de hansenologia. Bauru: Centro de Estudos "Dr. Reynaldo Quagliato"; 2000. Capítulo 8, Manifestações clínicas e reações; p. 51 8.

4 Kahawita IP, Walker SL, Lockwood DNJ. Leprosy type 1 reactions and erythema, nodosum leprosum. An Bra Dermatol. 2008 Jan-Feb;83(1):75-82.

5 Jopling WH, McDougall AC, Bakos L. Manual de hanseníase. 4. ed. Rio de Janeiro: Atheneu; 1991.
6 Foss NT. Episódios reacionais na hanseníase. Medicina. 2003 abr-dez;36(2/4):453-9.

7 Sociedade Brasileira de Hansenologia; Sociedade Brasileira de Dermatologia. Hanseníase: episódios reacionais - projeto e diretrizes. Belo Horizonte: Associação Médica Brasileira; 2003.

8 Ministério da Saúde (BR). Secretaria de Vigilância em Saúde. Sistema Nacional de Vigilância em Saúde: relatório da situação da hanseníase - Pará. 5. ed. Brasília: Ministério da Saúde; 2011 . (Série C. Projetos, programas e relatórios).

9 Lana FCF, Amaral EP, Lanza FM, Lima PL, Carvalho ACN, Diniz LG. Hanseníase em menores de 15 anos no Vale do Jequitinhonha, Minas Gerais, Brasil. Rev Bra Enferm. 2007 nov-dez;60(6):696-700.

10 Aquino MAV, Santos JS, Costa JML. Perfil dos pacientes com hanseníase em área hiperendêmica do Estado do Maranhão, 1991-1995. Cad Saude Publica. 2003 jan-fev; 19(1):119-25. 
11 Pimentel MIF. Neurites na hanseníase: significado de parametros clínicos e epidemiológicos na indução e agravamento das incapacidades físicas em pacientes multibacilares [tese]. Rio de Janeiro (RJ): Universidade Federal do Rio de Janeiro; 1998.

12 Penna G, Pinto LF, Soranz D, Glatt R. High incidence of diseases endemic to the Amazon region of Brazil, 2001-2006. Emerg Infect Dis. 2009 Apr; 15(4):62632.

13 Miranda MBS. Reações hansênicas: estudo comparativo com esquemas poliquimioterápicos no Distrito Federal [dissertação]. Brasília (DF): Universidade de Brasília; 2005.
14 Andrade VLG, Araújo AJG, Ignotti E, Sabroza P. Estudo da adesão ao tratamento da hanseníase no município de Duque de Caxias - Rio de Janeiro: abandonos ou abandonados? Hansen Int. 2001 jan-jun;26(1):23-30.

15 Silveira IR, Silva PR. As representações sociais do portador de hanseníase sobre a doença [dissertação]. Joaçaba (SC): Universidade do Oeste de Santa Catarina; 2006.

Recebido em / Received / Recibido en: 10/4/2014 Aceito em / Accepted / Aceito en: 13/2/2015 